\title{
El protocolo verbal interactivo en la disminución de dificultades en la enseñanza de indexación
}

The interactive verbal protocol in the reduction of the difficulties in teaching indexing

\author{
Mariângela Spotti Lopes Fujita (1), Franciele Marques Redígolo (2) \\ y Paula Regina Dal'Evedove (3)
}

(1) Universidad Estadual Paulista Júlio de Mesquita Filho - UNESP, Departamento de Ciencia de Información, Av. Hygino Muzzi Filho, 737, Campus Universitário, CEP. 17525900 - Marília, SP - Brasil, Caixa-Postal: 421, goldstar@flash.tv.br. (2) franbiblio@yahoo.com.br (3) dovesud@marilia.unesp.br

\begin{abstract}
Resumen
El objetivo del presente trabajo fue investigar el uso del Protocolo Verbal Interactivo por Pares de alumnos como recurso pedagógico con el propósito de disminuir las dificultades en la enseñanza de indexación de textos científicos. Las colectas de datos fueron realizadas mediante Protocolo Verbal Interactivo con pares de alumnos. La disminución de las dificultades pudo apreciarse en el desempeño de los dos alumnos aprendices en el proceso de lectura e indexación del artículo propuesto, así como también por el perfeccionamiento de las estrategias de asignación de los términos. La técnica del Protocolo Verbal Interactivo por pares resulta, por tanto, un recurso pedagógico eficaz en el aula y facilitó la evaluación y observación del desarrollo de la actividad de indexación de los indizadores aprendices.
\end{abstract}

Palabras clave: Lectura Documentaria. Indización. Protocolo Verbal Interactivo.

\section{Introducción}

El estudio de la lectura documentaria, realizada por el indizador con el objetivo de identificación de conceptos, necesita ofrecer asistencia sobre la relevancia del proceso de lectura y su influencia en el resultado de la indexación. La observación de la lectura documentaria se hace necesaria por que direcciona el desempeño de las demás operaciones involucrando la extracción de conceptos.

Los estudios de abordaje socio-cognitivo ofrecen importantes observaciones al respecto de la mente humana y su desarrollo en relación a la comprensión de lectura. Los estudios de perspectiva socio-cognitiva en Lectura Documentaria, como el de Fujita (2004), ofrecen indicaciones estratégicas de enseñanza que consideran el contexto como facilitador de la comprensión de lectura. El contexto, en la indexación, se relaciona con el conocimiento previo profesional

\begin{abstract}
The purpose of the research was to investigate the use of the Interactive Verbal Protocol by pairs of students as a pedagogical resource, aiming the reduction of the difficulties in teaching the indexing of scientific texts. The data collections were done with Interactive Verbal Protocol with pairs of students. The reduction of the difficulties could be noticed by the way in which both apprentice students acted concerning reading and indexing of the proposed article, and also in improving the strategies to asign terms. The technique of the Interactive Verbal Protocol by pairs has been introduced as a pedagogical resource in the classroom, and provides a tool for the evaluation and observation of the development of the indexing activity by apprentice indexers.
\end{abstract}

Keywords: Documentary reading. Indexing. Interactive Verbal Protocol.

que el indizador posee de experiencias anteriores, así como con el conocimiento sobre el sistema de información, sus políticas y objetivos.

Sobre este aspecto, Fujita, Nardi y Fagundes (2003) utilizaron técnicas introspectivas de colecta de datos, que consistían en observar los procesos mentales mediante la grabación de la exteriorización verbal de los sujetos durante la actividad de lectura orientada a la indexación, permitiendo la visualización de sus estrategias, procedimientos y dificultades.

En este sentido, se torna imprescindible la facilitación de oportunidades en la formación del indizador para que el proceso de cognición de la lectura documentaria sea conocido y desarrollado teniendo en cuenta los objetivos de la indexación.

De esa forma, la metodología de enseñanza de la lectura en el aula estará basada en las dificultades de lectura presentadas por los alumnos, 
asociando la teoría a la práctica de la lectura, lo que facilitará la comprensión. En este método, el alumno participante es un aprendiz incentivado a tratar al participante experimentado como un tutor personal y resolver las dudas siempre que existan dificultades en el desarrollo de la actividad propuesta. La técnica interactiva de la colecta de datos introspectivos proporciona a los participantes la posibilidad de desarrollar las actividades cotidianas de forma que el proceso sea observable por el profesor.

El trabajo que se relata tuvo como objetivo investigar el uso del Protocolo Verbal Interactivo por pares de alumnos como recurso pedagógico con el propósito de disminuir las dificultades en la enseñanza de indexación de textos científicos. En la modalidad del Protocolo Verbal Interactivo por Pares, observamos la interacción entre dos indizadores aprendices, el intercambio de informaciones en el proceso de formación de conocimiento, las dificultades y la disminución de las mismas.

Los resultados de esas aplicaciones del uso de la técnica introspectiva interactiva con pares de alumnos demostraron que se facilita el intercambio de experiencias y se acelera el proceso de enseñanza y aprendizaje de la metodología conforme a la perspectiva de interacción cognitiva. En el análisis de las dificultades y del proceso interactivo de aprendizaje para disminución de dificultades se utilizó la concepción de Zona de Desarrollo Proximal (ZDP) de Vygotsky (1978). Este proceso de superación de dificultades fue visto durante todo el desenvolvimiento de actividades de indexación y analizado durante la evolución de la actividad, de las discusiones entre los sujetos y de sus relatos. La zona de Desarrollo Proximal fue establecida por medio de la comparación del desenvolvimiento de los sujetos desde el inicio de la actividad, la discusión y posible solución de las dudas, de sus relatos positivos sobre la interacción, y del reflejo en el resultado de la indexación.

\section{El protocolo verbal interactivo en la enseñanza de indexación}

Según Fujita (2004, p.2), "la actitud del lector frente al texto, anteriormente vista como una recepción pasiva de mensajes, pasó a considerar el procesamiento mental de la información de la comprensión y evoluciona hacia una perspectiva de interacción entre el lector y el texto". Así, cuanto mayor sea la interacción entre las tres variables consideradas en la lectura -el lector, el texto y el contexto-, mayor y mejor será el nivel de comprensión. Entretanto, la complejidad y la subjetividad que envuelve la actividad de la indexación temática es determinada por la situación de cada variable, al relacionarse durante la lectura documentaria y producir dificultades características. El contexto, por ejemplo, es una variable presente y real para el indizador profesional que tiene en mente todo el conocimiento sobre el funcionamiento, la demanda y las reglas del sistema de información en la cual actúa. El mismo no actúa en el alumno de indexación que no tiene la vivencia de la actuación profesional $\mathrm{y}$, por lo tanto, no cuenta con el conocimiento previo del contexto. La falta de la variable contexto, sumada a la falta de familiaridad con textos de dominios específicos del conocimiento, amplifica las dificultades de la tarea de indexación en la sala de aula.

El conocimiento nuevo no es simplemente absorbido por la mente humana; sucede una fusión entre el conocimiento previo del lector, con la nueva información adquirida con la lectura (Rumelhart, 1977). El conocimiento previo es un elemento que se intitula esencial para el lector, durante el proceso de lectura, para que exista comprensión (Borba, 2003, p. 20).

Para Cavalcanti (1989) las estrategias de lectura son percibidas cuando hay una ruptura en la comprensión, entonces el lector desacelera la lectura y se torna metacognitivo. Para Fujita y Cervantes (2005), la cognición es una acción y el aprendizaje es una negociación entre el sistemas y el medio; si no ocurre interacción, no habrá cognición.

La técnica del protocolo verbal consiste en analizar todo el proceso de verbalización del participante mientras realiza sus actividades con el mínimo de interacción con el investigador. Esta exteriorización es grabada y transcrita literalmente, produciendo protocolos verbales. Según Cavalcanti (1989), protocolos son generalmente definidos como relatos verbales de los procesos mentales conscientes del informante, es decir, se refieren al "pensar alto" del informante mientras realiza una tarea de cualquier naturaleza.

El protocolo verbal permite la observación del proceso de lectura porque el lector verbaliza el conocimiento procesual que posee para el desarrollo de la actividad. El conocimiento procesual permite que la lectura sea consciente, que el lector perciba la forma en que el texto está siendo leído y los niveles de comprensión alcanzados por él. En este contexto, el protocolo verbal proporciona información sobre los pasos de procesamiento individual -tales como verbalizaciones espontáneas, secuencia de movimientos con los ojos-, exteriorizando sus procesos 
mentales y manteniendo la secuencia de las informaciones procesadas.

Al realizar ese tipo de protocolo con el mínimo de interacción con el investigador, el sujeto permanece imposibilitado de obtener mayores conocimientos, pues no existe intercambio de información, que podrían proporcionar una mejor reflexión y auxilio en los problemas que se van presentando.

La metodología de protocolo verbal individual, propuesta por Ericsson y Simon (1987), fue la base para la elaboración de dos nuevas metodologías que son el protocolo verbal en grupo y el protocolo verbal interactivo.

La metodología de Protocolo Verbal propuesta por Ericsson y Simon (1987) fue adaptada por Nardi (1999) en su investigación del papel del profesor como facilitador de la comprensión de metáforas en la lectura en lengua extranjera por universitarios de Biblioteconomía. El estudio de Nardi (1999) adaptó el Protocolo Verbal para la investigación con grupos de personas desarrollando eventos de lectura, colaborando para la observación de la cognición socialmente construida, denominándolo de Protocolo en grupo. Además del Protocolo en grupo para discusión de texto, se realizó observación participante con uso de protocolos verbales individuales y prácticas de lectura colaboradoras.

Según Nardi (1999), la observación participante de Spradley (1980) abarca niveles crecientes de participación: pasiva, moderada, activa y completa. En esta investigación, Nardi adoptó la observación participante moderada y con eso realizó una innovación en el uso de Protocolo Verbal que denominó "Protocolos Interactivos" o "Protocolos con escora", es decir, interactuó con el sujeto durante la lectura definiendo su participación como individuo experimentado. Nardo fundamentó este tipo de participación en la construcción de "escoras" de Palincsar (1986) basadas en la Zona de Desarrollo Proximal (ZDP) de Vygotsky.

La noción de ZDP utilizada en el análisis del protocolo verbal interactivo se basa en el desarrollo real y potencial del alumno. Vygotsky (1987, p. 22) define la Zona de Desarrollo Proximal (ZDP) como

[...] la distancia entre el nivel de desenvolvimiento real, que se acostumbra determinar a través de la solución independiente de problemas, y el nivel de desenvolvimiento potencial, determinado a través de la solución de problemas sobre la orientación de un adulto o en colaboración con compañeros más capaces.
La ZDP puede ser considerada como un espacio de intercambio de significados y sentidos (simbólico), entre el nivel de desarrollo real del aprendiz y el nivel de desarrollo potencial que puede alcanzar en interacciones con individuos más experimentados, donde el conocimiento circula y el aprendizaje sucede. Así, el interés es un análisis del proceso de interacción que posibilita la emergencia de la ZDP. La noción de la ZDP fue utilizada en esta investigación en el análisis de las transcripciones del protocolo verbal interactivo.

A partir de la innovación propuesta por Nardi (1999), se puede resaltar que es necesario no solamente adecuar contenidos programáticos, sino también introducir metodologías de enseñanza de lectura, como por ejemplo, el uso de la técnica del protocolo verbal interactivo como recurso pedagógico.

Después del desenvolvimiento de la actividad de lectura para indexación, tanto el profesor como el alumno pueden discutir puntos específicos de la actividad que no quedaron claros o que propiciaron mayor nivel de dificultades, además de conversar sobre el desempeño de cada uno y sobre la situación de colaboración que propició el desenvolvimiento del aprendizaje. Ese momento es considerado posterior al uso de protocolo y, por eso, es conocido como Entrevista Retrospectiva.

Haastrup (1987), lingüista, fue una de las pioneras en el uso de técnicas introspectivas para investigar el proceso de lectura, por ejemplo, la Entrevista Retrospectiva. En su investigación, intentó verificar cuales eran las fuentes de conocimiento usadas por aprendices de inglés de diferentes niveles, para inferir significado en palabras desconocidas. Utilizó las técnicas de "pair thinking aloud" (protocolo realizado por pares de lectores) y la entrevista retrospectiva individual (basada en los datos elicitados por el protocolo). Así, la autora estableció una taxonomia de las fuentes de conocimiento (Nardi, 1993).

Nardi (1993), también utilizó la técnica de la Entrevista Retrospectiva, caracterizándola como entrevista indirecta retrospectiva inmediata, utilizada inmediatamente después de la aplicación del protocolo verbal, con el objetivo de obtener datos adicionales espontáneos que pudieran dar continuidad al proceso observado. Además, utilizó la entrevista directa retrospectiva prorrogada, aplicada después de algunas horas, para elicitar la retrospección más consciente a fin de confirmar el análisis de los datos. 
A continuación, presentaremos las descripciones de los procedimientos de colecta de datos del protocolo verbal interactivo aplicado con pares de alumnos.

\section{Metodología}

EI Protocolo Verbal Interactivo por pares es una metodología para la observación e investigación de los procesos mentales entre dos sujetos que realizan una misma tarea, principalmente, de representación de la información y del uso de estrategias. Consiste en la grabación de la exteriorización verbal del pensamiento de dos sujetos durante la realización de una determinada tarea (Fujita, 2003).

Mediante esta modalidad de protocolo verbal observamos la interacción entre los dos indizadores aprendices en la disciplina de "Indexación" del Curso de Biblioteconomía de la UNESP - Campus de Marília (1), para identificar la posibilidad de intercambio de información durante el proceso de formación del conocimiento, las dificultades y la disminución de las dificultades en la realización de la actividad de indexación de un texto científico.

La metodología de aplicación del Protocolo Verbal Interactivo por pares se divide en tres diferentes etapas:

- Procedimientos anteriores a la colecta de datos: definición del universo de investigación, selección del texto-base y de los sujetos, conversación informal con los sujetos.

- Procedimientos durante la colecta de datos: familiarización con la tarea del "Pensar Alto", grabación del "pensar alto" durante la lectura del texto-base.

- Procedimientos posteriores a la colecta de datos: transcripciones literales de las grabaciones, procedimientos de análisis.

3.1. Procedimientos anteriores

a la sesión de colecta de datos

\section{Definición del universo de la investigación}

Las colectas de datos fueron realizadas en la UNESP - Facultad de Filosofía y Ciencias, Campus de Marilia.

\section{Selección de los individuos participantes}

Para las dos colectas de datos, contamos con la participación de cuatro alumnos de graduación, de tercer año de Biblioteconomía de la UNESP de Marília/SP, por estar cursando la disciplina de "Indexación" y haber tenido un primer contacto en la disciplina de "Lectura Documentaria".

\section{Definición de los papeles de la investigadora}

Los objetivos de la investigadora en la aplicación del Protocolo Verbal Interactivo en pares de alumnos fueron:

- Verificar las dificultades de los aprendices en la realización de la tarea de indexación.

- Analizar el papel del Protocolo Verbal Interactivo en pares como técnica de colecta de datos introspectivos y como recurso pedagógico.

- Observar la relación de interactividad entre los dos sujetos aprendices.

- Al término de la actividad de indexación realizada en conjunto por los propios alumnos, la investigadora realiza la entrevista retrospectiva, conforme Haastrup (1987) para facilitar la exteriorización de las dificultades, uso de procedimientos y estrategias.

- Grabar la actividad de "Pensar alto".

\section{Selección del texto base}

Para los dos Protocolos Verbales Interactivos por pares de alumnos, se escogió el mismo texto base, un artículo del área de Salud extraído de la Revista Pró-fono R. Atual Científica, v. 18 , n.2, 2006, intitulado: Succión en recién nacidos pré-término y estimulación de la succión, de los autores: Flávia Cristina Brisque Neiva; Cléa Rodrigues Leone.

\section{Análisis de la Tarea por el investigador}

La investigación tuvo como objetivo describir la evolución del padrón de succión y los efectos de la SNN en RNPT, adecuados para la edad de gestación (AIG), en función del avance de la IG corregida, considerando que los padrones de SNN y succión nutritiva (SN) se modifican en la medida en que ocurre la maduración de esta función a lo largo del tiempo. Se realizó en el nido anexo a la maternidad del HC - FMUSP. Los materiales y métodos utilizados en los ensayos de los recién nacidos pré-término fueron el chupón, dedo con guante y, también, el seno materno vacío. Los resultados mostraron de que con el avance de la IG corregida, existió un aumento de ocurrencia de las características succión iniciada fácilmente (SIF); cierre de labios, lengua y mandíbula; acanalamento de lengua; peristaltismo de lengua; fuerza de succión, volumen de leche ingerido por el tiempo total. En la discusión se destaca que el uso del dedo con guantes, además de haber aumentado la probabilidad de ocurrencia de varias características de la succión en relación al chupón, también tiene la ventaja de requerir la presencia obligatoria de la persona que realiza esta estimulación junto al RN durante este procedimiento. En conclusión, lo encontrado en este estudio muestra 
que, con el pasar del tiempo y el aumento de la IG corregida, el proceso de maduración para la capacidad de succión evoluciona (Neiva; Leone, 2006).

\section{Conversación Informal con los sujetos.}

Anteriormente a la técnica de Protocolo Verbal Interactivo en pares, se realizó una conversación informal con los participantes, mencionando los objetivos de la investigación y mostrando su importancia para el desenvolvimiento del área en Análisis Documentario. Los participantes fueron informados que sus identidades permanecerían ocultas en la transcripción de los datos, con el objetivo de dejarlos más confortables durante la realización de la tarea.

Se realizó también una familiarización con la tarea del "Think Aloud" - "Pensar Alto o Protocolo Verbal”, adaptado de Nardi (1993), cuyo propósito es presentar los procedimientos necesarios para auxiliar a los aprendices en el desempeño de la tarea.

Se solicitó que los sujetos realizaran la lectura del texto-base teniendo como objetivo la identificación de conceptos para la indexación.

\subsection{Procedimientos durante la sesión de colecta de datos}

Para el inicio del Protocolo Verbal Interactivo por pares de alumnos, se entregó el texto base a los sujetos aprendices que, a continuación, realizaron la actividad juntos, por medio de preguntas individuales e intercambio de comprensión.

La función de la investigadora, en el momento de la aplicación, fue controlar el grabador y recordar a los sujetos que exteriorizaran sus procesos mentales, el "pensar alto".

Los sujetos iniciaron la lectura del artículo científico utilizando su conocimiento previo, comenzaron realizando una lectura de las partes del artículo, con la intención de entender el asunto.

Al término de la actividad, solicitamos a los sujetos, en una conversación retrospectiva, que relataran cuales fueron sus mayores dudas; una visión de los participantes de la modalidad de Protocolo Verbal en pares; y también que indicaran posibles mejoras al instrumento de observación.

\subsection{Procedimientos después de la sesión de colecta de datos}

Se realizó la transcripción de los datos integralmente, siguiendo las anotaciones adaptadas de Cavalcanti (1989). Además de la identificación de las fuentes de las conversaciones individua- les de los sujetos aprendices en el Protocolo Verbal Interactivo por pares de alumnos, creamos categorías que abarcaran las dificultades de los sujetos. Después de destacar las categorías, regresamos a la transcripción de los datos para retirar las partes de las conversaciones que se encuadraban en cada categoría.

\subsection{Decisión sobre el análisis de los datos}

Los datos colectados fueron divididos en turnos, siguiendo la secuencia de la conversación de cada participante en la aplicación.

El análisis de los datos colectados consistió en la identificación de las dificultades de los aprendices en la indexación del artículo científico y de los relatos de superación de las dificultades y declaraciones de los aprendices sobre la actividad realizada, como forma de visualizar la zona de desenvolvimiento proximal (ZDP) del alumno, establecida por Vygotsky (1978).

\section{Resultados}

La técnica de colecta de datos Protocolo Verbal Interactivo por pares de alumnos permitió observar el desenvolvimiento de los sujetos en relación a los niveles más satisfactorios en la realización de la tarea de indexación, a partir de la dinámica de interacción entre los sujetos durante el análisis de asunto para la identificación y selección de conceptos del texto-base seleccionado, con el propósito de intercambiar experiencias y acelerar el proceso de aprendizaje.

La aplicación de la técnica ocurrió conforme perspectiva de interacción cognitiva, juntamente con el análisis y exploración de la Zona de Desenvolvimiento Proximal (ZDP) de los alumnos a fin de verificar las dificultades y la interacción cognitiva, la cual proporcionó la disminución de las dificultades y encaminó a los alumnos en el proceso de aprendizaje.

Esta modalidad de protocolo verbal posibilitó una mejor reflexión y auxilio sobre las dificultades presentadas durante el proceso de indexación a través de la comunicación continua entre los dos participantes.

Las dificultades detectadas aparecieron en la medida en que los sujetos entraban en conflicto en cuanto al entendimiento del texto-base y la identificación de términos para su posterior representación. Se obtuvieron resultados bastante diversificados entre los dos aprendices durante la realización de la indexación, destacándose como principales dificultades la falta de procedimientos de análisis de las partes estratégicas del artículo, es decir, de exploración de la estructura textual; el hecho de que el texto-base sea de una área de poco 
sea de una área de poco dominio de los sujetos; la presencia de términos específicos del vocabulario con los cuales los sujetos no poseían familiaridad y comprensión; la falta del apoyo de la experiencia profesional en lo que respecta al contacto inicial con la lectura documentaria en textos científicos; la falta de uniformidad en los procedimientos de análisis de asunto para la identificación de términos representativos; así como la dificultad inicial para la realización de la actividad por tratarse del primer contacto con la técnica de colecta de datos.

La disminución de las dificultades se presentó en el momento de la interacción entre los indizadores aprendices buscando el intercambio de información y la comprensión para la resolución de los problemas en cuestión.

El proceso de la superación de las dificultades es visualizado en el transcurso de todo el desarrollo de la actividad, por medio de la aplicación de la técnica del Protocolo Verbal Interactivo en pares. El siguiente ejemplo del relato realizado por uno de los sujetos permite una visualización clara de los beneficios y de la contribución proporcionada por el uso de esta técnica:

Con el método tú terminas entendiendo más el texto, reflexionando primero, para después identificar los términos.

Referente a la visión de los sujetos sobre la eficacia de la interacción se destacan los siguientes relatos:

Yo prefiero trabajar en dupla, porque son puntos de vista diferentes, en mi concepción puede estar correcto, pero en la del otro no, me parece interesante el trazarnos ideas.

Porque él puede tener informaciones que yo no sepa, y acresenta. Cuando permanecemos solos, si estamos con dudas, y si tuviéramos otra persona ella puede ayudarnos, ella puede saber y de repente resolver su duda.

Con el objetivo de destacar la interactividad ocurrida durante el proceso de indexación, seleccionamos la siguiente secuencia de conversaciones:

\section{"Sujeto 2}

La acción es una estimulación de la succión.

\section{Sujeto 1}

Será que son dos términos?

\section{Sujeto 1}

Entonces vamos a colocar en la acción la succión, por el momento.

\section{Sujeto 2}

Entonces si colocamos succión en el agente, y la nutrición en la acción?"

\section{Sujeto 1}

Entonces la acción es la estimulación..."

Partiendo de estas observaciones, se verificó el nivel de evolución y desenvolvimiento que los sujetos alcanzaron con la interacción, la cual permitió una mayor seguridad, agilidad y rapidez en la realización de la actividad en dupla a través de un auxilio mutuo de las partes con el objetivo de solucionar las dificultades y posibilitar la construcción del conocimiento.

Los resultados revelaron que el Protocolo Verbal Interactivo por pares de alumnos es un eficaz instrumento de colecta de datos, así como un excelente recurso pedagógico, pues debido a la interacción entre los indizadores aprendices las dificultades fueron discutidas y remediadas. De esta manera, se constató la funcionalidad de la técnica como relevante para el desarrollo del aprendizaje en el aula en lo que respecta a la actividad de indexación. Además, posibilitó observar el proceso de la lectura documentaria, lo que nos lleva a verificar su uso como recurso de aprendizaje para la enseñanza de estrategias que van a prevenir el contexto profesional en el abordaje socio-cognitivo, a través de actividades que coloquen al alumno en contacto con una situación real de trabajo.

\section{Consideraciones finales}

Considerándose la necesidad de perfeccionar la enseñanza de la indexación en la graduación, en este contexto, se verificó el uso del Protocolo Verbal Interactivo por pares de alumnos como una técnica de auxilio en la enseñanza de indizadores aprendices con objeto de promover la capacitación de los mismos frente a la actividad de indexación, donde la aplicación de la técnica fue muy satisfactoria y provechosa, pues posibilitó conocer el proceso de lectura documentaria realizada por los participantes de las colectas y la observación de la formulación de estrategias y dificultades encontradas por los aprendices.

A través del uso de Protocolo Verbal Interactivo por pares de alumnos como recurso pedagógico en disciplina de indexación, se tiene la expectativa de que la técnica sea de alta relevancia para los alumnos de graduación, posibilitando a los aprendices una capacidad de reflexión sobre la actividad de indexación que realizan, juntamente con la estimulación del uso de su conocimiento previo, de la observación y del desenvolvimiento de estrategias de lectura documentaria.

Por lo tanto, se constata que la interacción entre los participantes es de suma importancia en el desarrollo de la actividad y en el proceso de 
enseñanza y aprendizaje de indizadores aprendices.

De este modo, se recomienda la aplicación de la técnica del Protocolo Verbal Interactivo por pares de alumnos en el momento en que los indizadores aprendices demuestren algún tipo de dificultad para el desarrollo de la indexación, buscando promover el proceso de enseñanza y aprendizaje ocurrido por medio de la interacción entre los aprendices.

El desarrollo de los indizadores aprendices mediante el intercambio de comprensión y la realización conjunta de la tarea de indexación caracteriza la técnica como de fundamental importancia para la realización de la actividad de indexación de modo satisfactorio, además de traer confirmaciones sobre el proceso de enseñanza y aprendizaje.

Por medio de la técnica introspectiva de datos es posible observar el aprendizaje de los indizadores aprendices en el proceso de lectura documentaria, mediante la interacción de los participantes, y revelar dificultades en el proceso de enseñanza y aprendizaje. De esta manera, el Protocolo Verbal Interactivo por pares de alumnos actúa como un eficaz recurso pedagógico en el proceso de enseñanza de la lectura documentaria que estimula y promueve la formación del indizador aprendiz en el contexto de enseñanza y aprendizaje a partir de las experiencias de los alumnos en sala de aula; y viabiliza el aprendizaje por medio de la identificación de las dificultades presentadas por los aprendices y su desarrollo en dirección a los niveles más satisfactorios.

Resaltamos la efectiva viabilidad del Protocolo Verbal Interactivo por pares de alumnos en la enseñanza de indexación para indizadores aprendices, por ser un recurso pedagógico que permite la observación de la lectura documentaria de los indizadores de forma confiable para el análisis de los datos. Por lo tanto, la técnica actuó como reveladora de las principales dificultades presentadas durante el proceso de lectura realizado por los indizadores aprendices, motivo por el cual la consideramos un recurso de aprendizaje en la medida en que la exteriorización del pensamiento durante la lectura revela estrategias y dificultades que después de ser detectadas, podrán ser perfeccionadas.

\section{Notas}

(1) Curso de graduación en Biblioteconomía del Departamento de Ciencia de Información de la Facultad de Filosofía y Ciencias de la Universidad Estadual Paulista "Júlio de Mesquita Filho" -
UNESP - Campus de Marília - www.marilia. unesp.br.

(2) Mariângela Spotti Lopes Fujita: Profesora Adjunta del Departamento de Ciencia de Información de la Facultad de Filosofía y Ciencias de la Universidad Estadual Paulista - UNESP, Campus de Marília Brasil.

(3) Franciele Marques Redígolo: Alumna de Biblioteconomía de la Facultad de Filosofía y Ciencias de la Universidad Estadual Paulista - UNESP, Campus de Marília - Brasil, con beca otorgada por CNPq.

(4) Paula Regina Dal'Evedove: Alumna de Biblioteconomía de la Facultad de Filosofía y Ciencias de la Universidad Estadual Paulista - UNESP, Campus de Marília - Brasil, con beca otorgada por CNPq.

\section{Referencias}

Borba, E. A. (2003). Leitura para indexação: o uso da Linguagem Documentária como estratégia específica do leitor profissional. Marília: Faculdade de Filosofia e Ciências, UNESP, 2003. Trabalho de Conclusão de Curso.

Cavalcanti, M. C. (1989). I-n-t-e-r-a-ç-ã-o leitor-texto: aspectos de interpretação pragmática. Campinas: UNICAMP, 1989.

Ericsson, K. A., Simon, H. A. Verbal reports on thinking. (1987) // Faerch, C., Kasper, G. (eds.). Introspection in second language research. Clevedon: Multilingual Matters, 1987. 24-53.

Fujita, M. S. L. (2003). A leitura documentária do indexador: aspectos cognitivos e lingüísticos influentes na formação do leitor profissional. 2003. Marília: Faculdade de Filosofia e Ciências, UNESP, 2003. Tese de Livre-Docência em Análise Documentária e Linguagens Documentárias Alfabéticas

Fujita, M. S. L. (2004). A leitura documentária na formação inicial do indexador: a abordagem sócio-cognitiva na investigação de estratégias de ensino. Marília: Faculdade de Filosofia e Ciências, Universidade Estadual Paulista; CNPq. (Projeto Integrado de Pesquisa).

Fujita, M. S. L.; Cervantes, B. M. N. (2005). Abordagem Cognitiva do Protocolo Verbal na Confirmação de Termos para a Construção de Linguagem Documentária em Inteligência Competitiva. // Valentin, M. L. P. (org.). Métodos qualitativos de pesquisa em Ciência da Informação. São Paulo: Polis, 2005. 1: 29-57.

Fujita, M. S. L..; Nardi, M.I.A.; Fagundes, S.A. (2003). Observing documentary reading by verbal protocol. // Information Research, 8:4 (2003). http://informationr. net/ir/8-4/paper155.html. (2006-06-10).

Haastrup, K. (1987). Using thinking aloud and retrospection to uncover learners lexical inferencing procedures. II Faerch, C., Kasper, G. (eds.). Introspection in second language research. Clevedon: Multilingual Matters, 1987.

Nardi, M. I. A. (1993). As expressões metafóricas na compreensão do texto escrito em língua estrangeira. São Paulo: Pontifícia Universidade Católica, 1993. Dissertação de Mestrado.

Nardi, M. I. A. (1999). A metáfora e a prática de leitura como evento social: instrumentos do pensar a biblioteconomia do futuro. São Paulo: Pontifícia Universidade Católica de São Paulo, 1999. Tese de Doutorado. 
Neiva, F. C. B.; Leone, C. R. (2006). Sucking in preterm newborns and the sucking stimulation. // Pró-Fono R. Atual. Cient. Barueri, 18:2 (2006). http://www.scielo. br/scielo.php?script=sci_arttext\&pid=S0104-5687200600 0200003\&lng=en\&nrm=iso. $(2006-12-17)$.

Palinscar, A. S. (1986). The role of dialogue in providing scaffolding instruction. Educational Psychologist, 1986. Apud Nardi, M. I. A. (1999). A metáfora e a prática de leitura como evento social: instrumentos do pensar a biblioteconomia do futuro. São Paulo: Pontifícia Universidade Católica de São Paulo, 1999. Tese de Doutorado.
Rumelhart, D. E. (1997). Hacia uma comprensión de la comprensión. // Rodríguez, E., Lager, E. (org.). La lectura. Santiago de Cali: Editorial Universidad del Valle, 1997. p. 25-51.

Spradley, J. (1980). Participant observation. New York: Holt Hinehart \& Winston, 1980. Apud Nardi, M. I. A. (1999). A metáfora e a prática de leitura como evento social: instrumentos do pensar a biblioteconomia do futuro. São Paulo: Pontifícia Universidade Católica de São Paulo, 1999. Tese de Doutorado.

Vygotsky, L. S. (1987). Pensamento e Linguagem. São Paulo: Martins Fontes, 1987, p. 22-37. 